\title{
Artificial intelligence-based defect detection and classification using pulsed infrared thermography
}

\author{
by Yuxia Duan ${ }^{1}$, Hai Zhang ${ }^{2,3}$, Caiqi Hu¹, Shicai Liu ${ }^{1}$, Xavier Maldague ${ }^{2}$, Ning Tao ${ }^{4}$ \\ 1 Central South University, School of Physics and Electronics, 932 Lushan South Road, 410083, Changsha, \\ China.yuxia.duan@csu.edu.cn \\ 2 Université Laval, Department of Electrical and Computer Engineering, 1065, av. de la Médecine, G1V 0A6, \\ Québec (QC), Canada. \\ 3 University of Toronto, Department of Mechanical and Industrial Engineering, 5 King's College Road, M5S 3G8, \\ Toronto (OV), Canada. \\ 4 Capital Normal University, Physics Department, 105 West-Sanhuan North Road, 100048, Beijing, China.
}

\begin{abstract}
As artificial intelligent (AI) technology develops, neural networks, and more complex deep learning algorithms have come to be used in infrared thermography to detect and estimate defects. This study reports the results from automatic defect detection and classification methods that used a multilayer feed-forward neuron network (NN) model and a deep learning architecture: long short term memory recurrent neural network (LSTM-RNN).
\end{abstract}

\section{Introduction}

Infrared thermography, as a stand-alone, non-destructive testing (NDT) method has been used in many fields, including composite materials, printed circuit boards, buildings, and art [1]. Defect detection, and classification, are a concern during material fabrication and testing. Inspecting hollow honeycomb-structured materials, which are susceptible to liquid ingress is a typical example. A small amount of water ingress in an aircraft honeycomb structure is usually tolerable. But even a small amount of hydraulic oil in a rudder, wing, or other key components, could cause serious mechanical failure. Detecting and characterizing the type of fluid ingress in aircraft is critical for targeted maintenance.

As artificial intelligent $(\mathrm{Al})$ technology develops, neural networks, and more complex deep learning algorithms have come to be used in infrared thermography to detect and estimate defects. Neural network (NN), a method of machine learning, has been applied in many fields, including, data mining, pattern reorganization, and fault diagnosis. In this study, thermal signal reconstruction (TSR) coefficients were used as features in the constructed NN model [2]. LSTM-RNN as a state-of-the-art deep learning architecture specifically designed for time-series forecasting, was employed to automatically classify common defects occurring in honeycomb materials, including debonding, adhesive pooling, water, and hydraulic oil ingress. Temperature contrast values over time were used as input to train, and test the model.

\section{Methodologies}

Defect classification in infrared thermography is a problem of multi-class classification in machine learning. This paper presents, multilayer, feed-forward NN and LSTM-RNN models employed to classify defects.

Neural network (NN) is a product of simulation of how to manage information in the human brain. It is already known as strong tools in various fields, including, data mining, pattern reorganization, and fault diagnosis [3]. The input layer consists simply of feature vectors. The next layers are called hidden layers. The final layer is the output layer, where there is one node for each class. In the training phase, the correct value for each input is known, which is termed supervised training. The calculated output value is compared with the correct value to compute an error term for each node, which is then fed back through the network. These error terms are then used to adjust the weights in the hidden layers. After repeating this process for sufficient training cycles, hopefully, the output values will be closer to the correct values.

RNN is a state-of-the-art deep learning architecture specifically designed for time-series forecasting. RNN is now mainly used to deal with unsegmented recognition of handwriting and speech. While learning temporal sequences, prior learned patterns memory in RNN can fade over time. Hochreier et al. proposed LSTM to tackle the problem of gradient vanishing by creating paths where gradient flows for long durations [4]. The core idea behind LSTM architecture is the memory unit which keeps its state over time and stores useful information for an extended time. It then uses past information and some gates that contain save, write, and read functions, to control information flow into a network in order to select which information enters the cell.

\section{Results and Evaluation}

We have carried out multiple experiments on two specimens by optical pulsed thermography (PT). One specimen is made of homogenous stainless steel specimen. The other specimen is made of Nomex honeycomb material. 


\subsection{Flat-bottom holed Specimen}

Four optical pulsed thermography were carried out on the stainless steel specimen. In the first three, the four FBH were filled with the same substances: air, water, and oil, respectively. In the fourth experiment, the holes were filled with different substances. The thermal sequences obtained from the first three experiments were taken as training data. The data obtained from the fourth experiment was used for testing. The NN model of six coefficients as input features, which are obtained from TSR, is constructed as 6-30-15-4. The defect classification results of testing data are shown in Fig. 1.

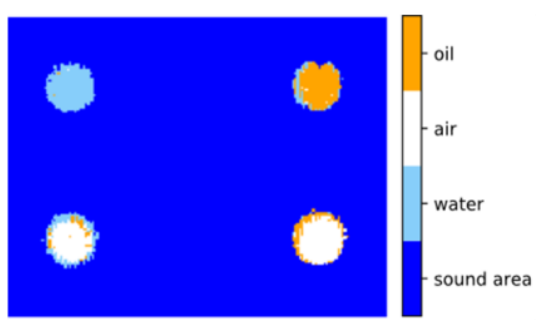

(a)

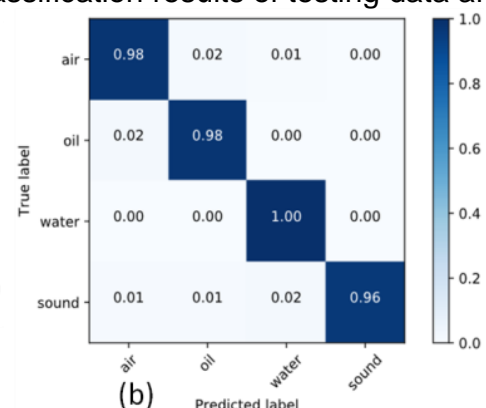

(b)

Fig. 1: Defect classification results of the stainless steel specimen using NN Model 6-30-15-4: (a) defect map; (b) the corresponding normalized confusion matrix (coefficients from TSR used as features).

\subsection{Honeycomb-structured specimen}

We extended our research to the classification of four kinds of defects in honeycomb-structured materials, including debonding, adhesive pooling, water, and hydraulic oil ingress. Two experiments were carried out on the specimen with pre-fabricated defects. In the first experiment, water and hydraulic oil were injected into seven adjacent cells. Each cell was filled with $120 \mu \mathrm{L}$ liquid. Optical pulsed-thermography was used to examine the test specimen. Thermal sequences obtained during the first experiment were used as training data. In the second experiment, three additional cells, for a total of ten, were filled with a liquid. First and second test experimental parameter settings were identical. The LSTM-RNN model is constructed, and the cooling curve of each pixel was used as an independent training/test data. The defect classification results of testing data are shown in Fig. 2.

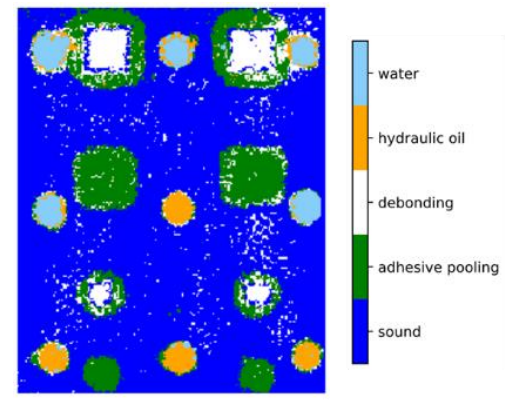

(a)

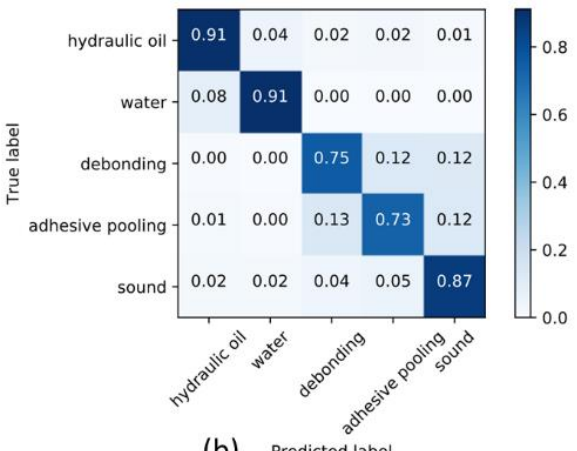

(b) Predicted label

Fig. 2: (a) Defect classification of the honeycomb-structured specimen using LSTM-RNN model: (a) defect map; (b) the corresponding normalized confusion matrix.

\section{Conclusions}

Automated classify defects by artificial intelligence is an attractive subject worldwide. The classification results indicated that LSTM-RNN model had satisfactory accuracy in classifying four kinds of defects in honeycomb-structured materials, including debonding, adhesive pooling, water, and hydraulic oil ingress. Artificial intelligence-based automated defect classification is feasible and has potential application in the field of targeted aviation maintenance.

\section{REFERENCES}

[1] Maldague XPV, Theory and practice of infrared technology for nondestructive testing, New York: Wiley, 2001.

[2] Shepard SM and Lhota JR, Reconstruction and enhancement of active thermographic image sequences, Optical Engineering, vol.45, 2003, pp.1337-1342.

[3] Awodele $\mathrm{O}$ and Jegede $\mathrm{O}$, Neural networks and its application in engineering, Science \& IT, 2013.

[4] Hochreiter S, Schmidhuber J. Long short-term memory. Neural computation vol. 9(8), 1997, pp.1735-1780. 